\title{
GENTRIFICACIÓN EN EL CONTEXTO DE LA SOCIEDAD DEL RIESGO: EL CASO DE BARRIO ITAlia en Santiago de Chile
}

Gentrification in the Context of Risk Society: The Case of Barrio Italia in Santiago de Chile

\section{Jonathan Uri Colodro Gotthelf} jucolodr@uc.cl

Centro de Desarrollo Urbano Sustentable, Chile

RESUMEN: La «latinogentrificación» corresponde a la expresión latinoamericana del proceso de gentrificación, asociado a la globalización y, por lo tanto, cada vez más común en los espacios urbanos. Uno de los casos más ilustrativos en la ciudad de Santiago de Chile lo constituye el Barrio Italia, produciéndose en un contexto neoliberal y con un definido proceso de gobernanza de actores.

El presente ensayo analiza la situación de la gentrificación del Barrio Italia, en el contexto de la Teoría de la Sociedad del Riesgo de Ulrich Beck y la expulsión de residentes originales, lo que iría en contra del Derecho a la Ciudad planteado por Henri Lefebvre.

Palabras ClaVe: gentrificación, riesgo, gobernanza, Derecho a la Ciudad.

ABSTRACT: Latinogentrification is the Latin American term for the gentrification process, associated with globalization and therefore, increasingly common in urban spaces. One of the most illustrative cases in Santiago de Chile is Barrio Italia, framed in a neoliberal context and with a marked process of stakeholder governance.

This essay analyzes the situation of gentrification in Barrio Italia, in the context of Ulrich Becks' risk society theory and the eviction of its original neighbors, which contradicts the right to the city proposed by Henri Lefebvre. 
KEYWORDs: gentrification, risk, governance, right to the city.

RESUM: La «latinogentrificació» correspon a l'expressió llatinoamericana del procés de gentrificació, associat a la globalització i, per tant, cada vegada més comú en els espais urbans. Un dels casos més il·lustratius a la ciutat de Santiago de Xile el constitueix el barri Itàlia, ja que es produeix en un context neoliberal i amb un definit procés de governança d'actors.

Aquest assaig analitza la situació de la gentrificació del barri Itàlia, en el context de la Teoria de la Societat del Risc d'Ulrich Beck i l'expulsió de residents originals, fet que aniria en contra del Dret a la Ciutat plantejat per Henri Lefebvre.

Paraules clau: gentrificació, risc, governança, Dret a la Ciutat.

\section{Introducción}

Las ciudades contemporáneas son espacios en continua transformación. Cristalizan en el territorio los distintos procesos sociales que ocurren en su interior, dando origen a un palimpsesto en donde se yuxtaponen elementos sólidos que reflejan cambios líquidos (Bauman, 1999). Localizado en la zona pericentral de la ciudad de Santiago de Chile, el Barrio Italia corresponde a un interesante laboratorio urbanístico que en los últimos años ha experimentado un marcado proceso de gentrificación a partir de la migración de residentes con un mayor poder adquisitivo. Dicha situación ha derivado en transformaciones físicas, espaciales y sociales, las cuales se fundan en diversas causas: la búsqueda de nuevos espacios de residencia por parte de personas de ingresos medio-altos, su cercanía a las principales fuentes laborales del sector terciario, las características arquitectónicas y patrimoniales de sus edificaciones y la alta dotación de comercio, servicios y proximidad a redes de transporte 
como los buses del sistema Transantiago y Metro de Santiago. Es así como se ha ido construyendo un riesgo de exclusión social de aquellos habitantes con menores ingresos, situación que se analiza desde la perspectiva de la Teoría de la Sociedad del Riesgo, desarrollada por Ulrich Beck (1986), constituyendo una pérdida de derechos ciudadanos y cristalizando los procesos descritos por Lefebvre (1968), a partir de la promoción, el acoso inmobiliario y empresarial, así como el desplazamiento de los pobres hacia una periferia simbólica.

\section{Gentrificación en el pericentro de Santiago de Chile: el caso de Barrio Italia}

La gentrificación (derivada de gentrification en inglés, y traducida como «elitización» en castellano) consiste en un proceso económico, social y espacial que afecta a los mercados urbanos, generando progresivos aumentos del precio de suelo (Smith y Williams, 1986). Dichas transformaciones han sido estudiadas en diversas ciudades del mundo, teniendo una expresión particular en áreas centrales y pericentrales, incluyendo además, una resignificación de los espacios, cuyo origen industrial o residencial, da un giro hacia una actividad comercial, ligada al ocio, el consumo y la producción artística y cultural, sumando además una transformación de clases sociales (Schlack y Turnbull, 2011; Wyly y Hammel, 1999).

Dicho fenómeno ha variado su expresión espacial, produciéndose de forma particular en Latinoamérica, donde se habla de la «latinogentrificación». Se asocia al surgimiento de una nueva clase media y una relación con el proceso de globalización, estrechándose los lazos entre el mundo público y privado y renovando los cascos históricos (Butler y Hamnett, 1994; Ciccolella, 1999; Lees et al., 2008). Asimismo, esta variación espacial tiene que ver con la evolución histórica de la ciudad latinoamericana, con el surgimiento de los condominios cerrados en la periferia como principal espacio de vivienda no sólo para las clases más acomodadas, sino también para la clase media. Las dificultades de desplazamiento, generan un fenómeno de retorno a los centros históricos, en la búsqueda de viviendas cercanas a las fuentes laborales del sector terciario (Bordieu, 1997; Borsdof et al., 2007). 
Desde la perspectiva de Lefebvre (1968, p. 42), uno de los actores más importantes del urbanismo son los promotores, quienes «conciben y realizan (actuaciones) para el mercado, con propósitos de lucro, y ello sin disimularlo. Lo nuevo y reciente es que ya no venden alojamientos o inmuebles, sino urbanismo. Con o sin ideología, el urbanismo se convierte en valor de cambio».

Los casos de gentrificación estudiados en Chile se concentran principalmente en su capital (Santiago) y en otras grandes conurbaciones de la Zona Central, como el caso de Valparaíso (Sabatini et al., 2013; Hidalgo, Borsdorf y San Martín, 2014; Vergara-Constella y Casellas, 2016). Asimismo, existen investigaciones llevadas a cabo en ciudades intermedias como Iquique (Contreras, 2015; Labbé et al., 2016) y Talca (Inzunza-Contardo, 2014; Inzulza-Contardo y Díaz, 2016; Letelier y Rasse, 2016).

Los procesos que enfrenta Valparaíso se explican principalmente por su consolidación como la ciudad que concentra en mayor medida a la población de más bajos ingresos dentro de la conurbación de la que forma parte. Sin embargo, por su valor patrimonial, algunos de los cerros que la componen han comenzado a explotar su potencial patrimonial, consolidándose como espacios turísticos y experimentando un fuerte incremento en el valor del suelo, una re-orientación de sus actividades y un recambio de habitantes por personas de mayores ingresos, así como la instalación de hoteles boutique, hostales, tiendas especializadas para los turistas, cafeterías, bares y restaurantes.

Talca ha sido un caso interesante de analizar tras el terremoto de 2010, donde la ciudad sufrió grandes destrucciones que se expresan en cerca de 7700 propiedades con daños (Inzulza-Contardo, 2014). Dicha liberación de solares abrió una oportunidad para que procesos de destrucción creativa (Schumpeter, 1976) se desataran en el territorio, promoviendo la densificación del centro y la construcción de torres de departamentos, las cuales finalmente apuntan a otros grupos socio-económicos, de mayores ingresos a los previamente existentes en la zona. Entre 2010 y 2014, se construyeron alrededor de 17 proyectos habitacionales de estas características, en una ciudad intermedia que no supera los 250000 habitantes (Inzulza-Contardo, 2014). Por último, de acuerdo con Letelier y Rasse (2016), se configuró un «riesgo de relocalización», dado que numerosas familias, por lo general de bajos ingresos, fueron expulsadas hacia la periferia, donde obtuvieron sus nuevas viviendas. Una 
situación similar es la que sucede en Iquique, donde investigaciones recientes concluyen que el terremoto de 2014 abrió oportunidades para el desplazamiento de la frontera de la gentrificación, así como el recambio de habitantes en antiguas poblaciones cercanas al centro de la ciudad (Labbé et al., 2016).

El contexto local de la gentrificación santiaguina se caracteriza principalmente por la renovación y densificación residencial. El caso más representativo corresponde al centro de Santiago, donde a partir de su depresión durante el último tercio del siglo xx, se propone el Plan de Repoblamiento, llevado a cabo desde 1992 (Casgrain y Janoschka, 2013). Así, a partir de la promoción estatal y la aplicación de subsidios otorgados directamente a los usuarios finales, se construyeron grandes torres de departamentos en barrios en los que anteriormente se asentaron casonas para grupos sociales diversos, así como grandes paños en los que hubo fábricas, talleres, bodegas y viviendas antiguas, muchas de ellas en mal estado tras el terremoto de 1985. Este tipo de gentrificación, aunque sin necesariamente la promoción estatal, se repite en otros casos, principalmente asociados a ejes de transporte como el Metro de Santiago. Son ilustrativos casos como la Gran Avenida en San Miguel, la avenida Independencia y la avenida Recoleta en las comunas homónimas, y la avenida Irarrázaval en Ñuñoa. Sin embargo, este tipo de fenómenos asociados más bien a la densificación y una renovación residencial, no incluyen otros procesos como la puesta en valor de los barrios o la generación de una subcultura urbana (Zukin 1995), que es lo que ocurre en el caso que se analiza en el presente trabajo.

Así, en esta línea se han realizado investigaciones en sectores como el barrio Yungay, al oeste del centro histórico (Contreras, 2011; Paulsen, 2014; Mardones, 2017), barrio Concha y Toro (Contreras y Venegas, 2016) y barrio Bellavista en Providencia y Recoleta (Schlack y Turnbull, 2009; Schlack y Turnbull, 2011; Inzulza-Contardo, 2016). A lo anterior, se suman otras investigaciones en curso en relación al proceso de gentrificación reciente que experimenta el barrio El Aguilucho, en la comuna de Providencia (Colodro, 2017). En torno a Barrio Italia, que corresponde al caso empírico analizado en el presente ensayo, destacan los trabajos de Schlack y Turnbull (2009), Schlack y Turnbull (2011), Casgrain y Janoschka (2013), Contreras y Venegas (2016) e Inzulza-Contardo (2016). 
El Barrio Italia corresponde a un espacio socialmente construido, más allá de sus límites físicos. Es compartido por los municipios de Providencia y Ñunoa, en el pericentro oriente ${ }^{1}$ de la ciudad de Santiago de Chile (fig. 1).

$\mathrm{Su}$ historia se remonta a finales del siglo XIX, cuando la ciudad de Santiago comenzó a expandirse hacia espacios rurales contiguos. De acuerdo con Schlack y Turnbull (2011), existen cuatro etapas que caracterizan sus transformaciones socio-territoriales. Un inicio destinado a vivienda para la clase media-alta, que en aquel entonces se encontraba en un proceso de migración fuera del centro histórico de la ciudad ${ }^{2}$ (Borsdof, 2003). Un segundo momento, en el que ocurren subdivisiones prediales, que dan origen a industrias, talleres y bodegas, que convivían con la clase media. Una tercera etapa, donde el Barrio Italia es colonizado por artistas, atraídos por la vida de barrio y tranquilidad encapsulada en un área muy céntrica de la ciudad. La cuarta y última etapa se hace evidente a partir del año 2005, con transformaciones físicas y un recambio de habitantes. Aparece un nuevo tipo de comercio, al mismo tiempo que las antiguas casonas han sido paulatinamente restauradas y reemplazadas por pequeñas galerías, dando origen a un espacio inspirado en la vida europea, a diferencia del fuerte proceso que ha caracterizado la dinámica santiaguina con el surgimiento de los centros comerciales cerrados o malls a partir de la década de los ochenta del siglo pasado (fig. 2).

1. El peri-centro de Santiago corresponde a toda aquella área localizada fuera de la comuna de Santiago (centro) y su vez dentro del anillo Américo Vespucio. Se compone de las comunas de Providencia, Ñuñoa, Macul, San Joaquín, San Miguel, Pedro Aguirre Cerda, Estación Central, Quinta Normal, Independencia y Recoleta. Se trata de áreas urbanizadas durante el primer tercio del siglo Xx, teniendo un marcado componente histórico en sus edificaciones y en las actividades que se realizan en el interior de sus barrios.

2. La ciudad de Santiago, fuertemente segregada socio-económicamente, concentra los sectores de residencia de las clases más acomodadas en las comunas de Vitacura, Las Condes, Lo Barnechea, La Reina, Providencia y Ñuñoa, que componen el denominado «Cono de Altos Ingresos». Dicho espacio, se conformó a partir de la migración de las clases medias-altas y altas desde el centro de la ciudad (barrios como Concha y Toro, Yungay, Lastarria, Dieciocho, la zona del Club Hípico, entre otros) durante el transcurso del siglo $\mathrm{xx}$.

Así, el «Cono de Altos Ingresos» aglomera los mayores precios de suelo en el Gran Santiago, así como la mayor superficie destinada a oficinas y actividades comerciales, principalmente en torno al eje de las avenidas Providencia y Apoquindo, que constituyen una continuación del centro histórico de Santiago, cumpliendo la función de centro financiero y de servicios. 


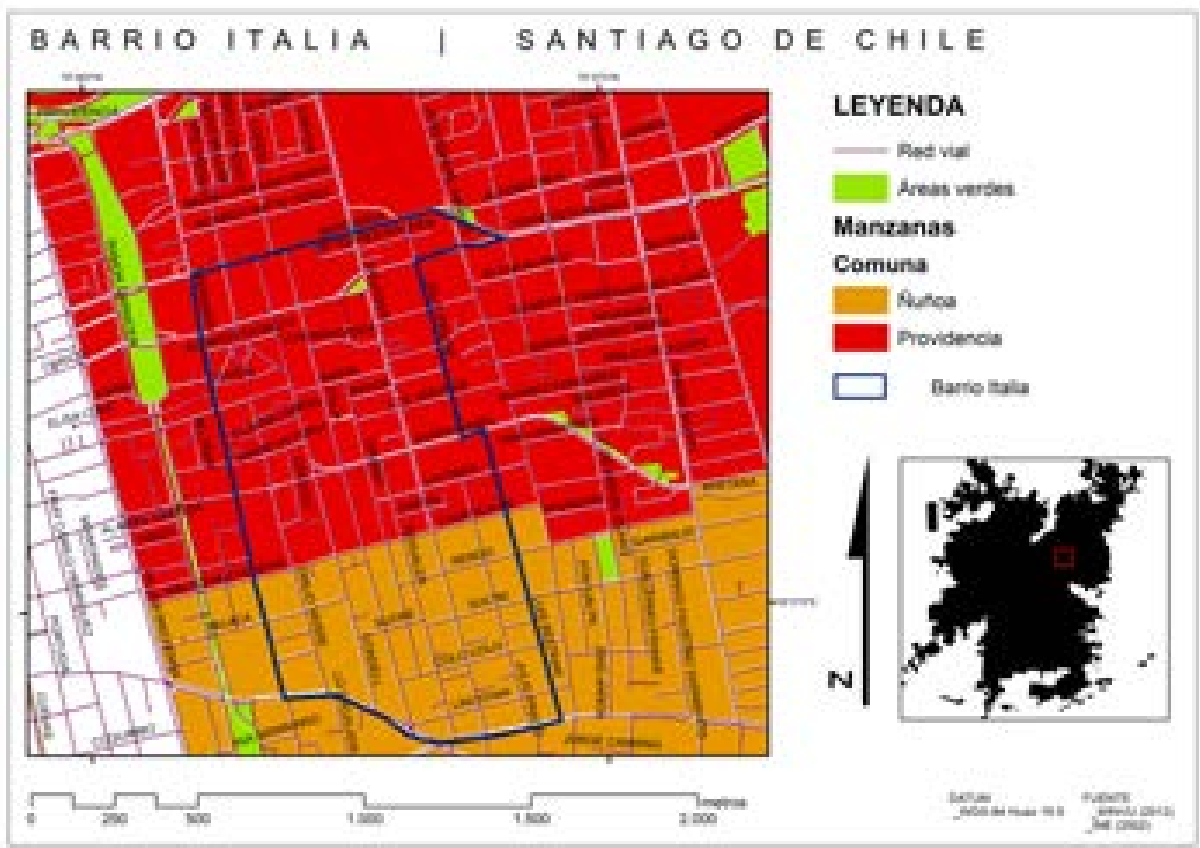

Figura 1. Localización del Barrio Italia en Santiago de Chile. Fuente: elaboración propia.

La gentrificación del Barrio Italia se trata de un proceso de reorientación de sus funciones, actividades y economía local. Si bien, el recambio de habitantes es posible de constatar a través de las diferencias entre los censos de 1992 y 2002 con un aumento considerable de los jefes de hogar clasificados en los grupos de más altos ingresos (véase el tercer apartado del presente artículo), lo más notable lo constituye la alteración del paisaje, así como el surgimiento de nuevas actividades comerciales.

La tipología de negocios que se asientan en el Barrio Italia es considerablemente diversa, pudiendo ser agrupados en tres grandes categorías. En primer lugar se encuentran los servicios más tradicionales, los cuales encapsulan cierta mística en el barrio, y que con el actual proceso de transformación, están en riesgo de desaparecer paulatinamente. Corresponden principalmente a antiguos bazares, ${ }^{3}$

3. El bazar corresponde a una tipología de comercio que se caracteriza por tratarse de una tienda de proximidad que vende distintos tipos de productos necesarios para la economía cotidiana del hogar. 
paqueterías, almacenes, talleres mecánicos y los tradicionales anticuarios característicos del barrio, dedicados a la fabricación y restauración de muebles. En segundo lugar están otros servicios posibles de encontrar en general en barrios santiaguinos como farmacias, supermercados, locales de comida al paso y algunas oficinas. En tercer lugar destacan los locales relevantes para el estudio de la gentrificación comercial. Este tipo de comercio es de carácter creativo y se vincula fuertemente a la existencia de artistas y determinados colectivos que habitan el barrio. Es aquí donde se clasifican las tiendas de ropa diseñada por conocidos autores de moda, muebles y decoración para el hogar, cafeterías innovadoras y de especialidad, bares de vinos chilenos, restaurantes chic y otras que llegan a ser extremadamente especializadas, dedicadas por ejemplo a la venta de productos de cuero no tradicionales, bolsos de moda, accesorios de cocina, entre otros numerosos rubros. Asimismo, se asientan firmas de publicidad, diseño, arquitectura y otras áreas profesionales creativas. Todos estos negocios se caracterizan por encontrarse principalmente en el interior de las galerías recientemente abiertas, a lo que se suman otras iniciativas como la restauración de grandes galpones en los que se han abierto hoteles boutiques, y el proyecto Factoría Italia, que recupera la fachada de un antiguo complejo fabril para instalar un centro comercial de grandes dimensiones y que incluiría tiendas de este tipo, consolidando al Barrio Italia como el distrito de diseño de la ciudad de Santiago.

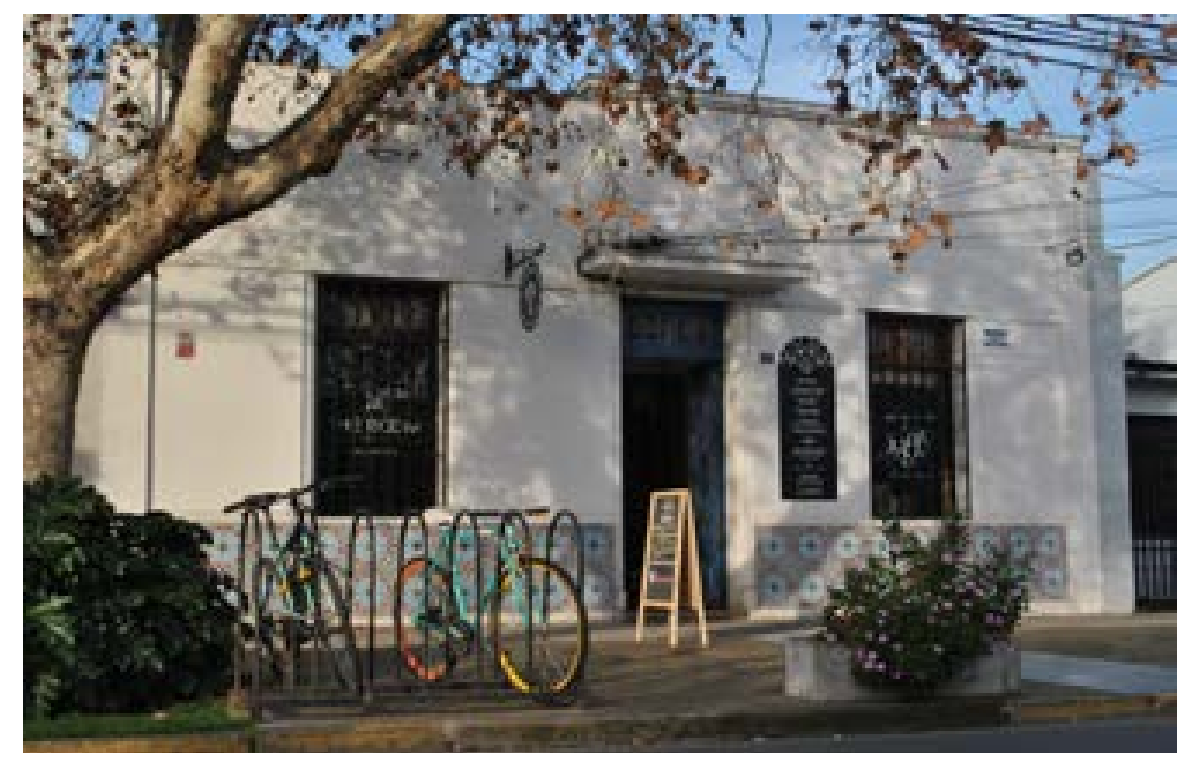

Figura 2. Galería Porta Nova en Barrio Italia. Fuente: elaboración propia. 


\section{Captura de plusvalías y Derecho a la Ciudad en el contexto de la Sociedad del Riesgo}

Los riesgos, de distinta naturaleza, son abordados desde la teoría de la Sociedad del Riesgo, propuesta por Ulrich Beck en 1986. Dicho abordaje comprende el riesgo como una producción social proveniente de la depredación de la naturaleza, la generación de nuevas amenazas y el deterioro de la cultura. Los riesgos son necesarios de minimizar, sin embargo, dicho proceso puede derivar tanto en nuevos riesgos como también en oportunidades en una sociedad capitalista. Así, hay una transformación del trabajo y la organización, las normas y la política (Beck, 1997). Se habla entonces, de «sociedad reflexiva», es un momento en que la sociedad se auto-cuestiona, ya que «desintegra y sustituye los supuestos culturales de las clases sociales por formas individualizadoras de la acción social» (Paulus, 2004, p. 12). El riesgo se asigna y reconoce socialmente, teniendo un contexto político y social, con una distribución inequitativa (Beck, 1986).

La modernidad reflexiva enseña los principios básicos de un cambio social en un marco estable. Dicho marco, sin embargo, está cambiando, ya que se produce «un nuevo tipo de capitalismo, un nuevo tipo de trabajo, un nuevo orden global, un nuevo tipo de sociedad, un nuevo tipo de subjetividad, un nuevo tipo de vida cotidiana y un nuevo tipo de Estado» (Beck, Bonss y Lau, 2003, p. 3). Este continuo cuestionamiento se expresa en las fuertes críticas que recibe el proceso de gentrificación y el surgimiento de movimientos de resistencia (Wacquant, 2008; López, 2011).

La idea descrita anteriormente recibe el nombre de «efecto boomerang», donde el riesgo vence las barreras de la riqueza. Se sustenta en que las interdependencias derivadas del proceso de globalización, finalmente, generan una transferencia de dichos riesgos, desde las sociedades menos desarrolladas hacia las de mayor desarrollo (Beck, 1986). Algunos de los riesgos se visibilizan, mientras que otros se intentan ocultar dependiendo de las necesidades y el papel que cobren los gobiernos con respecto a un caso determinado. Todo aquello que amenaza la vida, también amenazará al mercado (Beck, Bonss y Lau, 2003).

En el caso de la gentrificación, la colonización de nuevos residentes, y por lo tanto, un aumento de la demanda, puede derivar en un riesgo de exclusión 
social de los residentes originales, mermando su Derecho a la Ciudad. En este sentido, el suelo es un bien espacial, con tres peculiaridades: la sustituibilidad entre lotes urbanos con similitudes, la imposibilidad de reproducir la tierra, y la posibilidad de los empresarios de retener la oferta esperando mejores oportunidades de venta (Sabatini, 2000). La demanda de tierras en espacios que sufren un proceso de elitización tiene que ver con la obtención de plusvalías, las cuales se producen de manera escalonada y progresiva (López, 2011). Este mecanismo en inglés recibe el nombre de rent gap, donde cada uno de los agentes inversores pretende capturar la mayor plusvalía posible, vendiendo a un precio más alto que el de compra del inmueble, dando valor a su ubicación (Sabatini, 2000). Dicho valor se complementa también con otras características que presenta el barrio, el cual se consolida a través del tiempo con una imagen comercial. Como se aprecia en la figura 3, el barrio cuenta con una asociación de locatarios, quienes se preocupan de promoverlo a partir de una marca, a la cual se integran los distintos locales comerciales que cuentan con un determinado nivel y calidad del servicio que ofrecen.

En el caso de Santiago de Chile, se ha producido un fenómeno que ha cobrado especial fuerza desde el retorno de la democracia, en el cual los espacios pericentrales han sufrido lo que Schumpeter (1976) denomina como «destrucción creativa». Surgen nuevas oportunidades de inversión seguras, ya que es sabido que el precio del suelo seguirá aumentando en los años próximos, reemplazándose las antiguas viviendas por construcciones en altura (López, 2011). En el caso de aquellos territorios más demandados por sus características patrimoniales y por la experiencia espacial que ofrecen, el fenómeno descrito se acentúa, permitiendo la obtención de mayores plusvalías en un menor período de tiempo. En los distritos censales del Barrio Italia, se observa un aumento de un $720 \%$ del número de personas jefas de hogar identificadas como «directivos y profesionales» entre los censos de 1992 y 2002 (Casgrain y Janoschka, 2013). Esta situación ilustra los cambios en la composición social del barrio y una marcada renovación de su población residente. Independientemente de la existencia de desplazamiento, ocurre una apropiación desigual de la renta del suelo, capturada principalmente por los agentes inversionistas y en una medida casi insignificante por la población residente original (López, 2011). 
De acuerdo con lo anterior, el proceso de gentrificación en sí mismo constituye un conflicto de poder económico entre actores (Hamnett, 1991), generando polarización social y reestructuración económica, cambiando la composición social de los barrios y alterando fuertemente el paisaje (Maher, 1994; Filion, 1991). El riesgo se sitúa en la situación de que los residentes originales del barrio ven cada vez más limitada su capacidad de pago de los alquileres de sus viviendas, reduciéndose también la posibilidad a acceder a los cada vez más elitistas servicios que el barrio ofrece. A partir de ello, y de la fuerte demanda que recae sobre estas áreas, las personas de menos ingresos se ven presionadas a vender o dejar de alquilar sus viviendas y moverse a barrios con precios similares a los que pagaban originalmente, los cuales usualmente se localizan en áreas periféricas, más segregadas.

La gentrificación aparece en el plano no tecnológico de la sociedad del riesgo. Ofrece amenazas derivadas del propio proceso, construyendo el riesgo al obtener su producto con la vulnerabilidad económica de los habitantes originales de los barrios en cuestión. La sociedad reflexiva cobra un papel esencial, ya que el proceso de elitización es cuestionado desde los argumentos del derecho a la ciudad. La crítica apunta a que la gentrificación constituye una pérdida de derechos urbanos, mermando la posibilidad de que personas de bajos ingresos puedan acceder a barrios bien equipados y comunicados. Los excedentes para producir plusvalía aparecen de manera artificial, siendo impulsados por agentes públicos y privados, que se alían para obtener beneficios de ello y dar origen a un ciclo progresivo (Harvey, 2013).

El riesgo es percibido de manera diferente por las personas de acuerdo a su nivel educacional (Skaburskis, 2010), situación que se produce en un mismo territorio de manera gradual al desencadenarse un proceso de gentrificación. En este sentido, el riesgo de la exclusión social no se generaría exclusivamente en aquellas personas más vulnerables, sino que en cierto momento podría traspasarse también a grupos de ingresos medios. La conciencia de clases, por su parte, tiene que ver con un proceso de individualización (Rosa et al., 2014). La economía chilena, al regirse por un sistema neoliberal, hace que el conocimiento del riesgo sea más complejo. Si bien, es posible calcularlo, no se han realizado estudios al respecto. Tampoco existen gestiones por parte de las autoridades locales y metropolitanas por evitar la exclusión social 
derivada de la gentrificación, situación que ha tenido éxito en otros contextos. Entre los mecanismos están las subvenciones a arrendatarios antiguos y al alza de los precios, la provisión de fondos para organizar gremios barriales y el cobro diferenciado de un impuesto territorial y un impuesto al visitante (Altrock, 2003; Marinotti, 2008). Sin embargo, la realidad urbana de Chile es diferente, ya que la rent gap es capturada en mayor medida por los inversores privados. La administración pública recupera tan sólo una pequeña parte de las plusvalías en el largo plazo, a través del impuesto territorial (López, 2011).

La percepción del riesgo, en el caso de la gentrificación, es percibida en mayor medida por aquellas personas con un grado de vulnerabilidad económica que no les permitiría ser resilientes a la amenaza del aumento del valor de las propiedades. En este sentido, la problemática es imperceptible para otras capas de la sociedad, que ven el proceso de gentrificación como una actuación positiva, que en cierto sentido permite «recuperar barrios», a través del surgimiento de un nuevo comercio, mejoras en los espacios públicos, la restauración y valoración patrimonial.

El proceso en sí, se estructura a partir de una gobernanza, ya que implica auto-organización interinstitucional (Jessop, 1998), operando en redes que coordinan a los actores y dan origen a dependencias mutuas (Pierre y Peters, 2000). El caso del Barrio Italia es impulsado por diversos actores, con intereses diferentes que se pueden lograr mediante un propósito común: la producción de capital económico. Dicho capital puede provenir tanto de la captura de plusvalías como de la producción social del espacio, vendiendo urbanismo desde la perspectiva del marketing. No sólo se ofrece vivienda y comercio, sino también un estilo de vida, una experiencia de compra y un urbanismo de marketing (Lefebvre, 1968). Dichos actores provienen de mundos diversos: las municipalidades de Providencia y Ñuñoa desde la lógica de Estado subsidiario realizan actuaciones urbanísticas. Asimismo, promueven cambios o mejoras a los planes reguladores en busca de un desarrollo urbano más armonioso, con densificación en algunos sectores (principalmente en el perímetro perteneciente a Ñuñoa) y protección patrimonial en otros (en el área de Providencia). Los inversionistas, por su parte, son representados por las constructoras e inmobiliarias, que en su interés por capturar plusvalías ofrecen proyectos de mayor calidad, con un diseño más elaborado y alto están- 
dar. Los nuevos vecinos y locatarios se organizan para conservar la «vida de barrio» y potenciar la experiencia que ofrece el barrio. Por otro lado, surgen organizaciones gremiales que promocionan los servicios y la «experiencia» que ofrece esta área de la ciudad «recuperada» y producida socialmente (ver fig. 3). En algunos casos, existe organización para evitar la expulsión de los residentes originales; sin embargo, en un contexto neoliberal, se hace complicado existiendo una acumulación de capital progresiva a través de la promoción y consolidación del barrio.

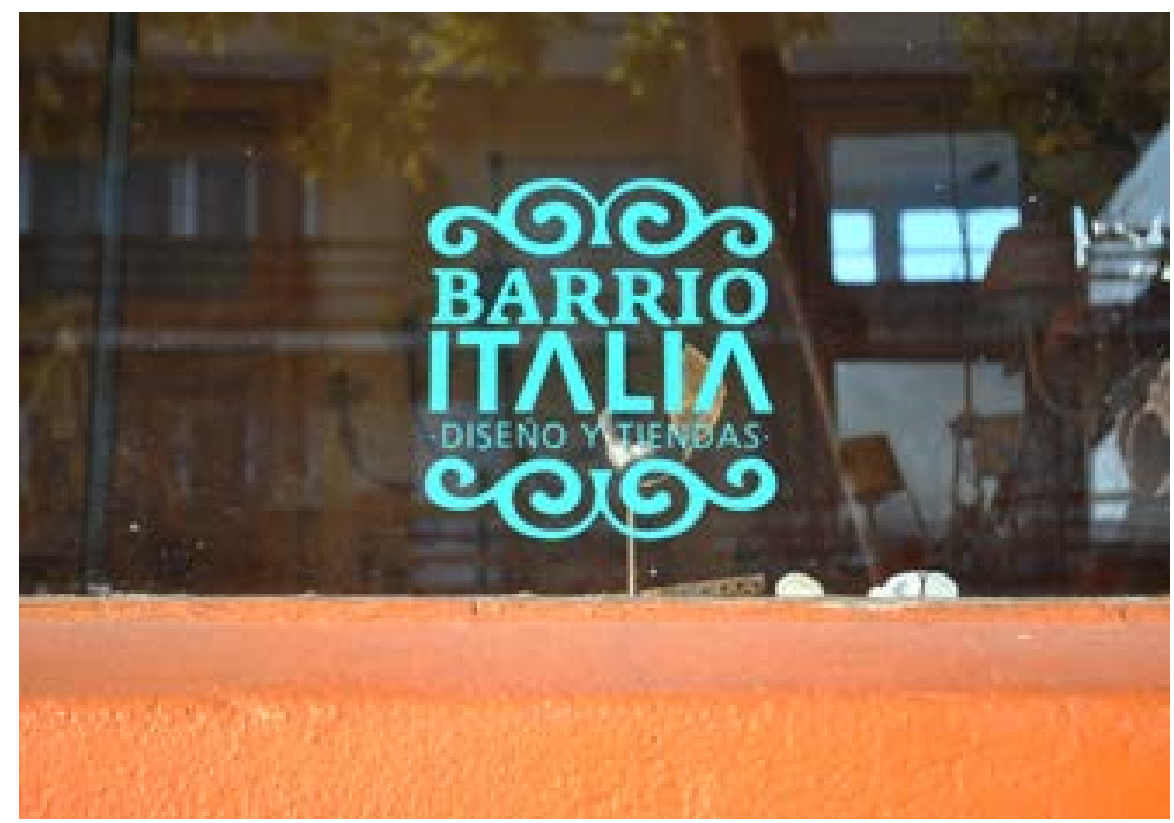

Figura 3. Impacto de las sociedades gremiales en la configuración del barrio como un espacio comercial auténtico. Fuente: elaboración propia.

\section{Conclusiones y reflexiones finales}

La gentrificación corresponde a un proceso que afecta a las ciudades en un contexto de globalización. En el caso de Chile, se da como «latinogentrificación», asociada a la protección patrimonial, la innovación, la producción cultural y la capitalización de lugares auténticos (Zukin 1995; Schlack y Turnbull, 2011). De ello, es posible que se produzcan procesos de gobernanza, que buscan maximizar la producción de capital para todos los actores que intervienen en el territorio, utilizando como mecanismo la captura de plusvalías. 
Sin embargo, el proceso descrito deriva en la exclusión social de los residentes originales. Ven reducida su capacidad de pago en un barrio cada vez más encarecido, generándose la construcción de un riesgo. Desde la perspectiva de Beck (1986) existe un conflicto económico y una percepción diferenciada del riesgo que se asocia fuertemente a lo que denomina «modernidad reflexiva».

La elitización es un proceso con efectos tanto positivos como negativos, ya que permite la protección patrimonial y el surgimiento de nuevas espacialidades. Sin embargo, involucra costos que son asumidos por los habitantes de menores ingresos, quienes transfieren beneficios a los actores que aprovechan la captura de plusvalías, dando origen a injusticias espaciales. El efecto boomerang se expresa de forma contraria a lo planteado por Beck (1986), ya que el riesgo es construido socialmente $\mathrm{y}$ transferido de forma directa, unidireccional y exclusivamente hacia aquellas personas más desposeídas. Sin embargo, con el aumento de la rent gap, dicho riesgo se va traspasando hacia capas superiores de la sociedad, hasta llegar a un punto en que el barrio quedará absolutamente elitizado y sólo asequible para actividades comerciales de alta rentabilidad y la residencia de personas de altos ingresos.

En el Barrio Italia, el proceso analizado está en pleno apogeo. Cada vez surgen nuevas oportunidades comerciales, así como la «colonización» de las calles interiores, donde todavía se cristalizan ciertos modos de vida tradicionales, así como la existencia de determinadas viviendas destinadas a personas de ingresos medio-bajos a través del sub-alquiler de habitaciones. En este sentido, es necesario para el mundo académico continuar monitoreando e investigando su evolución.

El mundo público, por su parte, tiene el desafío más grande, que corresponde en encontrar una fórmula que permita efectivamente la valorización del barrio y su conservación patrimonial, evitando la ruptura de los modos de vida tradicionales y la expulsión de los vecinos de menores ingresos. En el contexto neoliberal chileno, dicho desafío se profundiza, ya que el marco legal y la orientación de las políticas públicas operan de manera fragmentada y en general, con base en incentivos a la oferta y la demanda, careciendo de políticas integradas que permitan el desarrollo endógeno de los barrios, la detección temprana de procesos que pueden perjudicar a sus habitantes, así como la aplicación de acciones que permitan mitigar dichos procesos, o promover a que se generen de forma armónica y asistida por las administraciones locales.

Ejemplo de ello es la situación que enfrenta el espacio público de Barrio Italia, donde las aceras no se han adaptado adecuadamente al nuevo flujo de población que las utiliza para pasear y contemplar los escaparates de las tiendas, así como la instalación de terrazas de cafeterías y restaurantes. Asimismo, el espacio vial es aca- 
parado por vehículos que aparcan allí, sobre todo los fines de semana, momento en que el barrio recibe la mayor cantidad de visitantes.

\section{Agradecimientos}

Se agradece el apoyo del Centro de Desarrollo Urbano Sustentable (CEDEus), CONICYT/FONDAP $\mathrm{N}^{\circ} 15110020$ y a Pascuala Beckett, licenciada en Arquitectura de la Universidad de Chile, por su ayuda durante el trabajo de campo. Asimismo, se agradece al par evaluador anónimo por su valioso aporte y correcciones para mejorar la calidad del presente artículo.

\section{Referencias}

Altrock, U. (2003). «Aufwertung und Verdrängung. Versuch einer planungsethischen Positionsbestimmung zu einem Dauerbrenner in der Planung». En Altrock et ál. (eds), Jahrbuch der Stadterneuerung. Berlin: Tu Berlin.

Bauman, Z. (1999). Modernidad líquida y fragilidad humana. Buenos Aires: Fondo de Cultura Económica.

BEск, U. (1986). La sociedad del riesgo: hacia una nueva modernidad. Madrid: Paidós Ibérica.

- (1997). «La reinvención de la política: hacia una teoría de la modernización reflexiva. En U. Beck, A. Giddens y S. Lash. Modernización reflexiva. Política, tradición y estética en el orden social moderno. Madrid: Alianza.

Bonss, W. \& LAU, C. (2003). «The Theory of Reflexive Modernization: Problematic, Hypotheses and Research Programme». Theory, Culture \& Society, Vol. 20(2): 1-33.

Bordieu, P. (1997). Das Elend der Welt. Zeugnisse und Diagnosen alltäglichen Leidens an der Gesellschaft. Konstanz: Universitäts-Ver- lag Konstanz.

Borsdorf, A. (2003). «Cómo modelar el desarrollo y la dinámica de la ciudad latinoamericana». Eure, 29 (86), pp. 37-49.

Hidalgo, R., y Sánchez, R. (2007). «A New Model of Urban Develop- ment in Latin America: The Gated Communities and Fenced Cities in the Metropolitan Areas of Santiago de Chile and Valparaíso». Cities, 24, Vol. 5, pp. 335-398. 
Butler, T., \& Hamnett, C. (1994). «Gentrification, Class and Gender: Some Comments on Warde's Gentrification as Consumption». Environment and Planning: Society and Space, $\mathrm{N}^{\circ} 12$, pp. 477-493.

Casgrain, A., \& JanoschKa, M. (2013). «Gentrificación y resistencia en las ciudades latinoamericanas. El ejemplo de Santiago de Chile». Andamios. Revista de Investigación Social. Vol. 10, 22, pp. 19-44.

Ciccolella, P. (1999). «Globalización y dualización en la Región Metropolitana de Buenos Aires: grandes inversiones y reestructuración socioterritorial en los años noventa». EURE, Vol. 76, 25, pp. 5-27.

Colodro, U. (2017). «Nuevos territorios para la capitalización de lugares auténticos y la elitización: el caso del barrio El Aguilucho en Santiago de Chile». Anales de la Sociedad Chilena de Ciencias Geográficas [En prensa].

Contreras, Y. (2011). «La recuperación urbana y residencial del centro de Santiago: Nuevos habitantes, cambios socioespaciales significativos», EURE, Vol. 37(112), pp. 89-113.

Filion, P. (1991). «The Gentrification Social Structure Dialectic: A Toronto Case Study». International Journal of Urban and Regional Research. 15, pp. 553-573.

Galleguillos, X. (2014). «Latino gentrificación y polarización: transformaciones socioespaciales en barrios pericentrales y periféricos de Santiago, Chile». Revista de Geografía Norte Grande, 58, pp. 135-159.

HaCkworth, J. (2002). «Postrecession gentrification in New York City». Urban Affairs Review 37, pp. 815-843.

Hamnett, C. (1991). «The Blind Men and the Elephant: the Explanation of Gentrification». Transactions of the Institute of British Geographers, New Series, 16, pp. 173-189

Harvey, D. (2013). Ciudades Rebeldes: Del Derecho de la ciudad a la revolución urbana. Madrid: Akal.

Hidalgo, R., Borsdorf, A. \& SAn Martín, G. (2013). «Socio-spatial Chante in the World Heritage Site Valparaíso». Journal of the Geographical Society of Berlin, Vol. 144, 3. pp. 228-240.

Inzulza-Contardo, J. (2016). «Contemporary Latin American Gentrification? Young Urban Professionals Discovering Historic Neighbourhoods». Urban Geography, 37:8, pp. 1195-1214.

Inzulza-Contardo, J., \& Díaz, I. (2016). «Desastres naturales, destrucción creativa y gentrificación: estudio de casos comparados en Sevilla (España), Ciudad de México (México) y Talca (Chile)». Revista de Geografia Norte Grande, 64, pp. 109-128. 
- (2014). «La recuperación del diseño cívico como reconstrucción de lo local en la ciudad intermedia: el caso de Talca, Chile». Revista AUS, 15 , pp. 4-8.

Jessop, B. (1998). «Theory of Governance and the Risks of Failure: the Case of Economic Development». International Social Science Journal, 50, 155, pp. 29-45.

Labbé, G., Palma, P., Venegas, V., \& Ulloa, F. (2014). «Estigma territorial y gentrificación post desastre 2014. El caso de las poblaciones pericentrales norte de Iquique». Revista de Urbanismo, 34, pp. 34-54.

Lees, L., Slater, T., \& Wyly, E. (2008). Gentrification. New York y London: Routledge.

Lefebvre, H. (1968). El derecho a la ciudad. Barcelona: Península.

Letelier, F., \& Rasse, A. (2016). «Política de reconstrucción y desplazamiento: el caso de las familias de bajos ingresos del centro de Talca». Revista de Urbanismo, 35, pp. 220-245.

López, E. (2011). «Gentrification by Ground rent Dispossession: The Shadows Cast by Large-scale Urban Renewal in Santiago de Chile». Urban Studies, Vol. 35, 2, pp. 330-357.

López-Morales, E. (2013). «Gentrificación en Chile: aportes conceptuales y evidencias para una discusión necesaria». Revista de Geografia Norte Grande, 56, pp. 31-52.

Maher, C. (1994). «Housing Prices and Geographical Scale: Australian Cities in the 1980s». Urban Studies, 31, pp. 5-27.

Mardones, G. (2017). «El proceso histórico de la gentrificación en barrios “patrimoniales". El caso de barrio Yungay, Santiago de Chile». En Guérin, M. y F. Álvarez (eds.), Historia Sociocultural de América Latina. Espacio, migraciones y tiempo. Buenos Aires: Miguel Guérin.

Martinotti, G. (2008). «Lo que el viento se llevó. Espacios públicos en la metrópolis de tercera generación». En M. Degen y M. García (eds.), La metaciudad: Barcelona. Transformación de una metrópolis. Barcelona: Anthropos.

Paulsen, A. (2014). «Negocios inmobiliarios, cambio socioespacial y contestación ciudadana en Santiago Poniente. El caso del barrio Yungay: 2000-2013». En R. Hidalgo \& M. Janoschka (eds.), La ciudad neoliberal. Santiago de Chile: Pontificia Universidad Católica de Chile.

Paulus, N. (2004). «Del Concepto de Riesgo: Conceptualización del Riesgo en Luhmann y Beck». Revista Mad, 10. Paper 7.

Piere, J., \& Peters, G. (2000). Governances, Politics and the State. London: MacMillan Press. 
Rosa, E. ET AL. (2014). The Risk Society Revisited: Social Governance. Philadelphia, PA: Temple University Press.

SAbatini, F. (2000). «Reforma de los mercados de suelo en Santiago, Chile: efectos sobre los precios de la tierra y la segregación residencial». EURE, Vol. 26, 77.

Sabatini, F., Forno, S., Mora, P. \& Bustos, M. (2013). «Valparaíso: cerros de gente, cultura de diversidad». En F. Sabatini, G. Wormald, A. Rasse \& M. P. Trebilcock (eds.), Cultura de cohesión e integración social en ciudades chilenas, pp. 174-204. Santiago, Chile: Colección Estudios Urbanos Uc.

Schlack, E., \& Turnbull, N. (2009). «La colonización de barrios céntricos por artistas». Revista 180, (24), 2-5.

Schlack, E. \& Turnbull, N. (2011). «Capitalizando lugares auténticos: artistas y emprendimientos en la regeneración urbana». $A R Q, 79 . \mathrm{pp}$. 28-36.

SChumpeter, J. A. (1976). Capitalism, Socialism and Democracy. London: Routledge.

Skaburskis, A. (2010). "Gentrification in the Context of "Risk Society"». Environment and Planning, Vol. 42, pp. 895-912.

Smith, N. \& Williams, P. (1986). Gentrification of the City. London: Routledge.

Venegas, V. (2016). «Entre la consolidación y la latencia: gentrificación en barrios centrales de Santiago». Cuadernos de Vivienda y Urbanismo, 9(18), pp. 194-211.

Vergara-Constella, C., \& Casellas, A. (2016). «Políticas estatales y transformación urbana: ¿hacia un proceso de gentrificación en Valparaíso, Chile?». EURE, Vol. 42, 126, pp. 123-144.

Wacouant, L. (2008). «Relocating Gentrification: The Working Class, Science and the State in Recent Urban Research». International Journal of Urban and Regional Research. Vol. 32, 1, pp. 198-205.

Wyly, E. K., \& HAMmel, D. J. (1999). «Islands of Decay in Seas of Renewal: Housing Policy and the Resurgence of Gentrification». Housing Policy Debate, 10(4), pp. 711-771.

Zukin, S. (1995). The Culture of Cities. uK: Blackwell. 